\title{
Manipulating quantum-well states by surface alloying: Pb on ultrathin Ag films
}

\author{
T. Hirahara, ${ }^{1, *}$ T. Komorida, ${ }^{1}$ A. Sato, ${ }^{1}$ G. Bihlmayer, ${ }^{2}$ E. V. Chulkov,${ }^{3,4}$ K. He,${ }^{5}$ I. Matsuda,${ }^{5}$ and S. Hasegawa ${ }^{1}$ \\ ${ }^{1}$ Department of Physics, University of Tokyo, 7-3-1 Hongo, Bunkyo-ku, Tokyo 113-0033, Japan \\ ${ }^{2}$ Institut für Festkörperforschung, Forschungszentrum Jülich, D-52425 Jülich, Germany \\ ${ }^{3}$ Donostia International Physics Center (DIPC), 20018 Donostia-San Sebastián, Spain \\ ${ }^{4}$ Departamento de Física de Materiales and Centro Mixto CSIC-UPV/EHU, Facultad de Ciencias Químicas, \\ UPV/EHU, Apdo 1072, 20080 San Sebastián, Spain \\ ${ }^{5}$ Institute for Solid State Physics, University of Tokyo, 5-1-5, Kashiwanoha, Kashiwa, Chiba 277-8581, Japan
}

(Received 1 May 2008; revised manuscript received 6 June 2008; published 3 July 2008)

\begin{abstract}
The electronic structure of ultrathin Ag films with 1/3 monolayer (ML) of $\mathrm{Pb}$ alloyed at the surface was investigated by angle-resolved photoemission spectroscopy. Compared to clean ultrathin Ag films, the energy positions of the quantum-well states (QWSs) moved closer to the Fermi level due to the change of the potential barrier at the film/vacuum interface. We found that the parabolic band dispersion of the QWSs become disturbed where they cross the surface-state bands, and furthermore, they were shown to follow the periodicity that is only present at the surface. Our results suggest that it is possible to tune the properties of the QWSs whose thickness is more than $10 \mathrm{ML}$ just by submonolayer deposition of heteroelements at the surface.
\end{abstract}

DOI: 10.1103/PhysRevB.78.035408 PACS number(s): 68.35. - p, 73.20. $-\mathrm{r}, 79.60 .-\mathrm{i}, 71.18 .+\mathrm{y}$

\section{INTRODUCTION}

Electrons in a thin metal film can be confined in the thickness direction and form discrete energy levels, the so-called quantum-well states (QWSs). Over the past years, many experiments have been conducted to directly probe the energy levels with angle-resolved photoemission spectroscopy (ARPES) ${ }^{1-7}$ scanning tunneling microscopy/spectroscopy, ${ }^{8,9}$ or investigate the thickness-dependent oscillation in the film characteristics due to the change in the number of Fermilevel crossings of the QWSs. ${ }^{10-12}$ Furthermore, intriguing phenomena due to the interaction of the QWSs with the underlying substrate have been reported ${ }^{4,13-16}$ and it is now even possible to manipulate the band dispersion of the QWSs by growing the films on an anisotropic substrate. ${ }^{17,18}$ These works tuned the QWSs by changing the film/substrate interface.

On the other hand, there are also surface states on ultrathin metal films and their interaction with the QWSs have been reported. ${ }^{19}$ At the surface, the inversion symmetry is broken and due to the so-called Rashba effect, the surfacestate bands become spin-split. ${ }^{20,21}$ This splitting was also found to influence the spin properties of the QWSs. ${ }^{21}$ For the surface states of clean $\operatorname{Ag}(111)$, it was shown that the splitting is too small to be observed experimentally because Ag is a light element. However, recently, Ast et al. have shown that by alloying $\mathrm{Ag}$ with heavy elements such as $\mathrm{Bi}$ or $\mathrm{Pb}$, the dispersion of the surface-state bands change drastically and they show giant spin-splitting. ${ }^{22}$ They explained this in terms of the in-plane potential gradient that arises due to the alloying. This experiment was done on a single crystal Ag surface, but it can be easily expected that this alloying will also influence the properties of the QWSs if we use an ultrathin Ag film.

In this paper, we have investigated the electronic structure of ultrathin $\mathrm{Ag}$ films grown on $\mathrm{Si}(111)-7 \times 7$ alloying only the surface with $\mathrm{Pb}(\mathrm{Ag}(111) \sqrt{3} \times \sqrt{3}-\mathrm{Pb}$ films $)$ using ARPES. This means that in contrast to the previous studies, we have manipulated the film/vacuum interface. We found that the energy positions of the QWSs move closer to the Fermi level compared to those of the clean $\operatorname{Ag}(111) 1 \times 1$ films, which can be explained by the potential barrier change at the film/vacuum interface, inducing change in the phase shift. Furthermore, the QWSs were shown to follow the $\sqrt{3}$ $\times \sqrt{3}$ periodicity that is only present at the surface. This can be explained either by an initial-state (umklapp reflection at the $\mathrm{Ag}$ /vacuum interface) or a final-state (umklapp scattering in the photoemission process) effect. We also found indication of interaction between the surface and quantum-well state bands where they cross each other. These results suggest that it is possible to tune the properties of the QWSs just by depositing submonolayer of $\mathrm{Pb}$ atoms on the surface. We also discuss the Rashba splitting of the surface-state bands of the alloy surface.

\section{EXPERIMENTAL AND CALCULATION METHODS}

An $n$-type $\operatorname{Si}(111)$ wafer ( $P$-doped, $1-10 \Omega \mathrm{cm})$ was used as the substrate. First a clean $\mathrm{Si}(111)-7 \times 7$ surface was prepared by a cycle of in situ resistive heat treatments. Ag was deposited on the $7 \times 7$ surface at $\sim 170 \mathrm{~K}$ using an alumina-coated tungsten basket. After deposition, the films were annealed back to room temperature. The reflection high-energy electron diffraction (RHEED) patterns showed a sharp $1 \times 1$ pattern after this procedure, with strong peaks corresponding to QWSs detected by ARPES. In this paper 1 monolayer (ML) is defined as the atom density in the $\mathrm{Ag}(111)$ plane $\left(1 \mathrm{ML}=1.39 \times 10^{15} \mathrm{atoms} / \mathrm{cm}^{2}, 2.36 \AA\right.$ thick). $\mathrm{Pb}$ was deposited on this $\mathrm{Ag}(111) 1 \times 1$ surface at $\sim 500 \mathrm{~K}$. After $\sim 0.2 \mathrm{ML}$ of $\mathrm{Pb}$ deposition, $\sqrt{3} \times \sqrt{3}$ spots were observed and the best surfaces were obtained at $\sim 0.33$ ML deposition as confirmed by the sharpness of the RHEED patterns as well as the strong intensity of the surface-state spectra. $^{23}$ The deposition rate of Ag was $0.50 \pm 0.05 \mathrm{ML} / \mathrm{min}$ as estimated by the completion of the $\mathrm{Si}(111) \sqrt{3} \times \sqrt{3}-\mathrm{Ag}$ superstructure formed at one silicon 

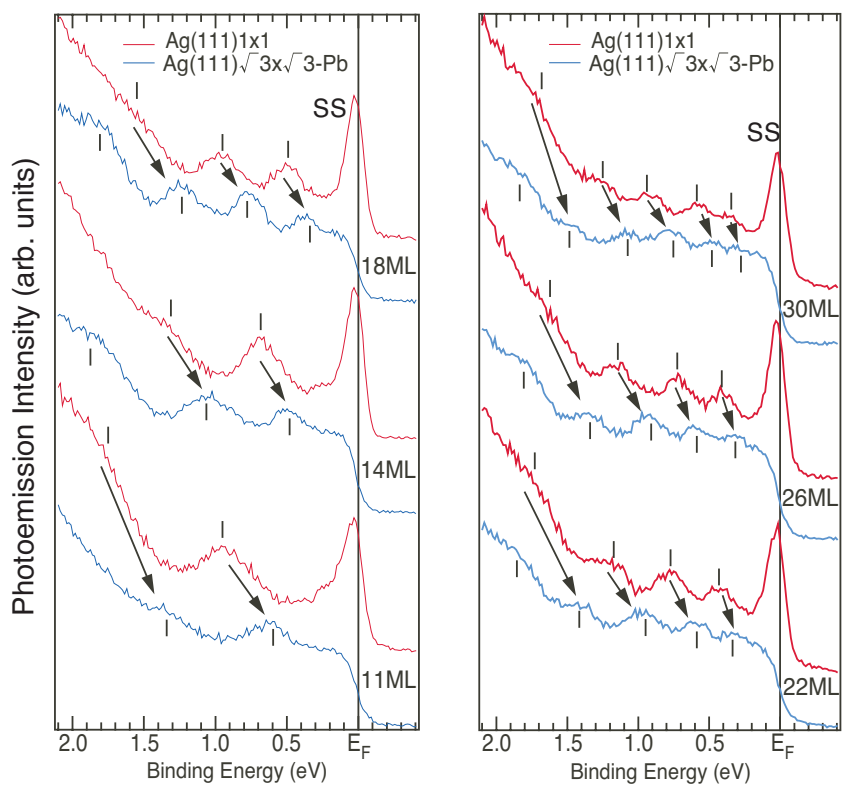

FIG. 1. (Color online) Normal-emission ARPES spectra for clean $\operatorname{Ag}(111) 1 \times 1$ [red (light gray) lines] and $\operatorname{Ag}(111) \sqrt{3}$ $\times \sqrt{3}-\mathrm{Pb}$ [blue (dark gray) lines] ultrathin films for various thicknesses. The peak positions are marked and the change due to $\mathrm{Pb}$ deposition is indicated by the arrow.

monolayer $\left(7.83 \times 10^{14}\right.$ atoms $\left./ \mathrm{cm}^{2}\right) .{ }^{24}$ The deposition rate of $\mathrm{Pb}$ was $0.06 \pm 0.01 \mathrm{ML} / \mathrm{min}$ as calibrated by the change in the phase from the $\mathrm{Si}(111) \beta \sqrt{3} \times \sqrt{3}-\mathrm{Pb}$ surface to the $\operatorname{Si}(111) \alpha \sqrt{3} \times \sqrt{3}-\mathrm{Pb}$ surface. ${ }^{25}$

ARPES experiments were performed with a commercial hemispherical photoelectron spectrometer (VG ADES-400) using unpolarized $\mathrm{HeI} \alpha(21.2 \mathrm{eV})$ radiation at room temperature. The Fermi level position $\left(E_{F}\right)$ was determined by measuring the metallic Fermi edge of a Ta foil fixed on the sample holder.

$A b$ initio calculations have been performed using the fullpotential linearized augmented plane wave (FLAPW) method in film geometry as implemented in the FLEUR program and generalized gradient approximation for the description of exchange-correlation potential. Spin-orbit coupling (SOC) was included in the self-consistent calculation as described in Ref. 26. A ten-layer film embedded in vacuum was used in the calculation. Two $\mathrm{Ag}$ and one $\mathrm{Pb}$ atoms were placed on one side of the surface to simulate the $\sqrt{3} \times \sqrt{3}$ structure [Figs. 2(e) and 2(f)]. The details can be found in Ref. 27.

\section{RESULTS AND DISCUSSION}

\section{A. Normal-emission spectra}

Figure 1 shows the ARPES spectra taken at normal emission for various thicknesses for the clean $\operatorname{Ag}(111) 1 \times 1$ ultrathin films [red (light gray) lines] and those after the $\mathrm{Pb}$ deposition $[\mathrm{Ag}(111) \sqrt{3} \times \sqrt{3}-\mathrm{Pb}$ ultrathin films, blue (dark gray) lines]. For the clean $\operatorname{Ag}(111) 1 \times 1$ films, there is a large peak just near $E_{F}$ which corresponds to the surface state (SS). At higher binding energies, we see that the number of peaks changes systematically with film thickness, which is a typical behavior found in metal films and they can be assigned as QWSs. ${ }^{2,3}$ For the $\mathrm{Pb}$-adsorbed films, we notice that the large surface-state peaks found for the clean Ag films have vanished. Furthermore, we also find that the energy positions of the QWSs become closer to the Fermi level as indicated by the arrows. This means that just by adding $1 / 3$ $\mathrm{ML}$ of $\mathrm{Pb}$ onto Ag films whose thicknesses are more than 10 ML, we can tune the energy positions of the QWSs.

In order to elucidate the origin of the shift of the energy level of the QWSs, we apply the conventional phase-shift accumulation (PSA) model ${ }^{4,28}$ often used in analyses of QWSs. ${ }^{1,2}$ In this model, the condition that a QWS is formed is expressed by the Bohr-Sommerfeld quantization condition: ${ }^{1}$

$$
\phi_{\text {sub }}(E)+\phi_{\text {vac }}(E)+2 k_{\perp \text { env }} d=2 \pi(n-1),
$$

where $\phi_{\text {sub }}, \phi_{\text {vac }}$ are the reflection phase shifts at the film/ substrate and the film/vacuum interfaces, respectively, $k_{\perp \text { env }}$ is the wave number of the envelope function of a Bloch state perpendicular to the surface, $d$ is the thickness of the film, and $n$ is the quantum number. The wave number of the envelope function $k_{\perp \text { env }}$ is the wave number measured from the Brillouin zone boundary ( $L$ point in this case). Finding QWS peaks at the same energy $(E)$ for different thicknesses $\left(d, d^{\prime}\right)$ (quantum numbers $n, n^{\prime}$ ) allows one to derive $k_{\perp \text { env }}$ as

$$
k_{\perp \text { env }}(E)=\frac{n^{\prime}-n}{d^{\prime}-d} \pi .
$$

Figure 2(a) shows the dispersion $E\left(k_{\perp \text { env }}\right)$ determined in this way. ${ }^{29}$ The red open circles show the dispersion obtained from the QWSs of the clean $\operatorname{Ag}(111)$ films and the blue filled circles are the ones for the $\mathrm{Pb}$-adsorbed films. We fitted these data points with a function based on the two-band nearlyfree-electron model:

$$
E\left(k_{\perp \text { env }}\right)=E_{0}-\left\{a k_{\perp \text { env }}^{2}+U / 2-\left[4 a^{2} b k_{\perp \text { env }}^{2}+(U / 2)^{2}\right]^{1 / 2}\right\},
$$

where $a=h^{2} /\left(8 \pi^{2} m^{*}\right), b=3 \pi^{2} / a_{0}^{2}\left(a_{0}\right.$ is the lattice constant of Ag), $U=4.2 \mathrm{eV}$ (the width of the band gap at the $L$ point), and $E_{0}=0.31 \mathrm{eV}$ (the position of the $s p$ band edge relative to $\left.E_{F}\right) .5,6$ The solid black line shows the result of the fit and the fitting parameter $m^{*}$ yields $0.73 m_{e}$, which is consistent with previous reports on $\mathrm{Ag}$ films. ${ }^{2,5,6}$ Then using the fitting curve and Eq. (1), we can extract the total phase shift $\Phi_{\text {tot }}(E)$ $=\phi_{\mathrm{sub}}+\phi_{\mathrm{vac}}$ for each QWS as a function of the binding energy from the following equation:

$$
\Phi_{\mathrm{tot}}(E)=2 \pi(n-1)-2 k_{\perp \text { env }}(E) d .
$$

Figure 2(b) shows the calculated values of the total phase shift. The red open and blue filled circles show the data for the clean $\operatorname{Ag}(111)$ and $\operatorname{Ag}(111) \sqrt{3} \times \sqrt{3}-\mathrm{Pb}$ films, respectively. The solid lines show the results of a fit to a straight line. ${ }^{7}$ We see that $\Phi_{\text {tot }}$ is quite different for the two films; the energy dependence seems to be reversed. Thus we can say from this analyses that the change of the phase shift is responsible for the shift of the energy position. Because there should basically be no change at the film/substrate interface 

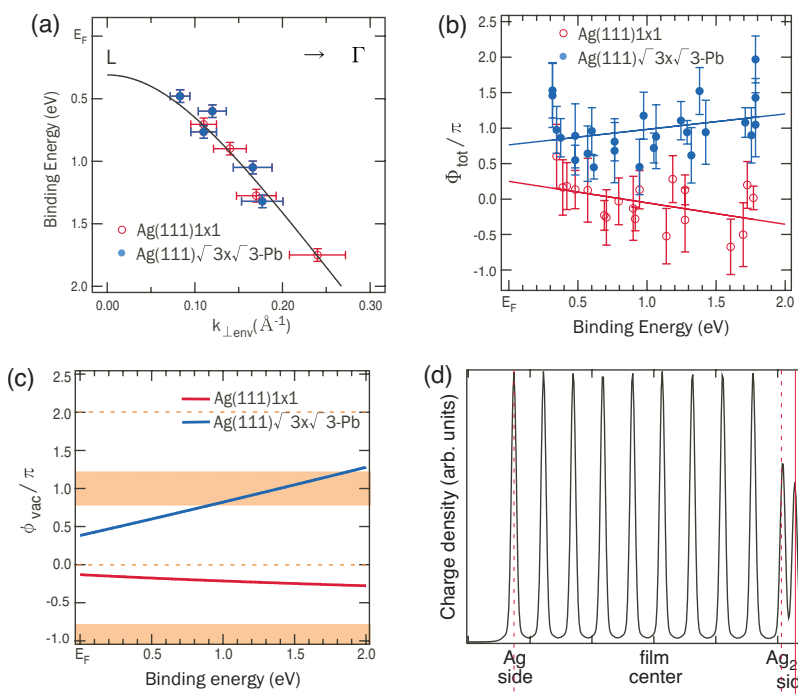

(e)
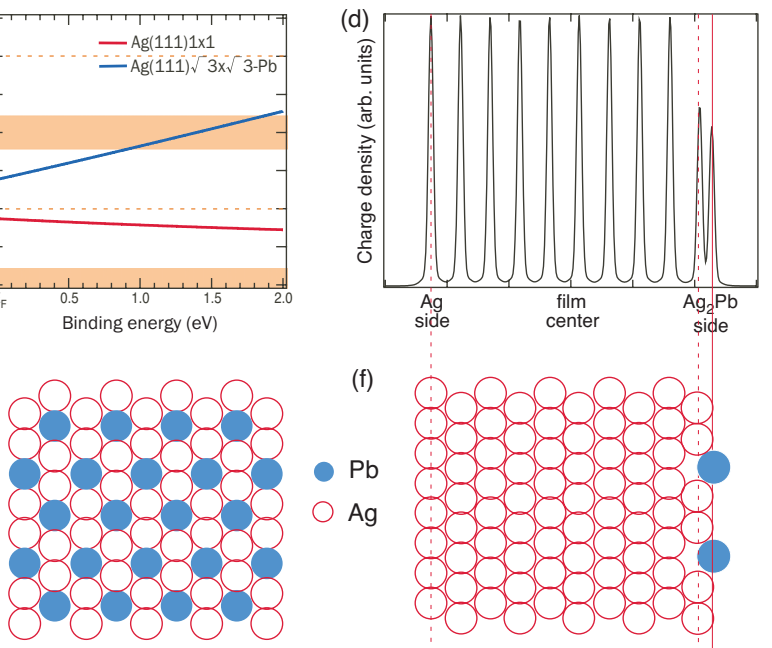

(f)

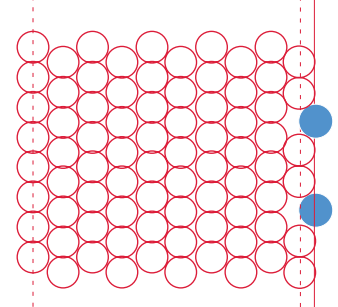

FIG. 2. (Color online) (a) The $s p$-band dispersion for Ag bulk along the $\Gamma-L$ Brillouin zone line. The red (open) and blue (solid) circles are data points from the present experiment obtained from the clean $\operatorname{Ag}(111) 1 \times 1$ and $\operatorname{Ag}(111) \sqrt{3} \times \sqrt{3}-\mathrm{Pb}$, respectively. The solid curve is the least-squares fit based on the two-band nearlyfree-electron model (see text). (b) Energy dependence of the total phase shift for the clean $\operatorname{Ag}(111) 1 \times 1$ (red open circles) and $\operatorname{Ag}(111) \sqrt{3} \times \sqrt{3}-\mathrm{Pb}$ (blue solid circles) films. The solid lines are the least-squares fit of the respective data. (c) Energy dependence of the phase shift at the film/vacuum interface for the clean $\operatorname{Ag}(111) 1$ $\times 1$ [red (light gray)] and $\mathrm{Ag}(111) \sqrt{3} \times \sqrt{3}-\mathrm{Pb}$ [blue (dark gray)] films. (d) The charge density distribution for the $10 \mathrm{ML} \mathrm{Ag} \mathrm{slab}$ which one side is alloyed with $\mathrm{Pb}$. For the $\mathrm{Ag}_{2} \mathrm{~Pb}$ side, the charge spill-out into the vacuum is reduced. The solid (dotted) line shows the position of the outermost $\mathrm{Pb}(\mathrm{Ag})$ layer. $(\mathrm{e}, \mathrm{f})$ Schematic drawing of the Ag slab used in the calculation viewed from the top (e) and the side (f), respectively.

$\phi_{\text {sub }}$, this difference can be attributed to $\phi_{\text {vac }}$.

For $\phi_{\text {vac }}$, the phase shift for an image potential within the WKB approximation ${ }^{30}$ is often used at the metal/vacuum interface for nonalloyed metal films. ${ }^{2,3}$ It can be expressed as

$$
\phi_{\mathrm{vac}}(E)=\pi\left(\frac{3.4}{E_{v}-E}\right)^{1 / 2}-\pi,
$$

where $E_{v}$ is the vacuum energy level. The results for the $\mathrm{Ag}$ (111) surface is shown in Fig. 2(c) by the red (light gray) line using the work function of $4.5 \mathrm{eV}$. Then $\phi_{\text {sub }}$ is obtained as a function of the binding energy by combining the results in Figs. 2(b) and 2(c) for the clean $\operatorname{Ag}(111)$ film. Finally, from the linear fits of Fig. 2(b), $\phi_{\text {vac }}$ for the $\operatorname{Ag}(111) \sqrt{3}$ $\times \sqrt{3}-\mathrm{Pb}$ films can be estimated assuming that $\phi_{\text {sub }}$ is the same between the clean and $\mathrm{Pb}$-adsorbed films. The result is

presented in Fig. 2(c) by the blue (dark gray) line. We notice that $\phi_{\text {vac }}$ for the $\operatorname{Ag}(111) \sqrt{3} \times \sqrt{3}-\mathrm{Pb}$ films is close to the value of $\pi$, which is expected for a strong confinement (Bragg reflection). This finding suggests that by $\mathrm{Pb}$ adsorption, the potential barrier to confine the electrons has become very high and the charge spilling into the vacuum has decreased.

This anticipation can in fact be confirmed by looking at the energy-integrated charge density distribution inside the films. Figure 2(d) shows the result of the $a b$ initio calculation for the $10 \mathrm{ML} \mathrm{Ag}$ slab with one side terminated with $\mathrm{Ag}_{2} \mathrm{~Pb}$ to simulate the alloy, as schematically illustrated in Figs. 2(e) and 2(f) for the top and the side views, respectively. This structure model is supported by experimental techniques such as electron diffraction, transmission electron microscopy, and surface x-ray diffraction. ${ }^{31-33}$ As can be easily imagined, the charge is distributed at each Ag layer. Near the film/vacuum interfaces, we notice some difference between the two sides. For the $\mathrm{Ag}_{2} \mathrm{~Pb}$ side, there are two peaks corresponding to the $\mathrm{Ag}$ and $\mathrm{Pb}$ layers because in the calculation the position of $\mathrm{Pb}$ atoms are a bit located out into the vacuum due to relaxation, indicated by the red dotted and solid lines, respectively. ${ }^{27}$ Figure 2 (d) indeed shows that the charge that is just near the vacuum for the $\mathrm{Ag}_{2} \mathrm{~Pb}$ side (the charge density at the outermost part of the film of the $\mathrm{Ag}_{2} \mathrm{~Pb}$ side) has decreased compared to the clean $\mathrm{Ag}$ side, consistent with the expectation of less charge spilling mentioned above. So in summary, we conclude that due to $\mathrm{Pb}$ adsorption, the potential barrier changes dramatically and the charge spilling into the vacuum decreases, meaning that the confinement effect becomes stronger. This results in the change of the phase shift which changes the energy position of the QWSs. This strong potential barrier, in other words, a strong potential gradient normal to the surface, may be one of the origins of the large Rashba splitting observed for the surface-state bands. ${ }^{34,35}$

\section{B. In-plane dispersion}

We now turn to the in-plane band dispersion of the $\operatorname{Ag}(111) \sqrt{3} \times \sqrt{3}-\mathrm{Pb}$ ultrathin surface alloy films. Figure 3(a) shows the ARPES spectra for the $16 \mathrm{ML}$ film along the $\bar{\Gamma}-\bar{K}$ direction, and Figs. 3(b) and 3(c) show those for the $24 \mathrm{ML}$ film along the $\bar{\Gamma}-\bar{M}-\bar{\Gamma}$ direction. The high-symmetry points are those for the $\sqrt{3} \times \sqrt{3}$ surface Brillouin zone. To visualize the band dispersion more clearly, Figs. 4(a) and 4(c) show the $E-k$ diagram by taking the second derivatives of the original spectra in Figs. 3(a)-3(c), respectively. This procedure enhances the spectral features and the intensity scales linearly from black (minimum), green, to dark blue (maximum). We have confirmed that the energy positions of the bands are the same between the images and the corresponding raw spectra. In addition to the parabolic band dispersion of the QWSs found in $\operatorname{Ag}(111)$ films, ${ }^{2}$ we find other states that disperse downwards from the $\bar{\Gamma}$ point in both directions, shown by the solid lines. They are surface states with no thickness dependence as can be noticed by comparing Figs. 4(a) and 4(b) that show the same band dispersion for different thicknesses. In fact, these states have been observed in 


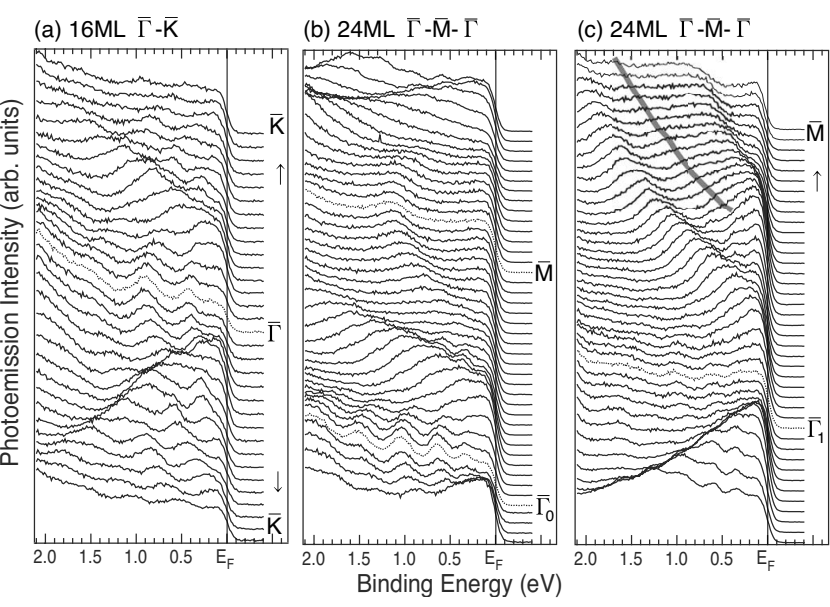

FIG. 3. ARPES spectra for the $16 \mathrm{ML}(\bar{\Gamma}-\bar{K}$ direction) (a), and the $24 \mathrm{ML}(\bar{\Gamma}-\bar{M}-\bar{\Gamma}$ direction) (b) and (c), for the $\operatorname{Ag}(111) \sqrt{3}$ $\times \sqrt{3}-\mathrm{Pb}$ films, respectively. Each spectrum was recorded in one degree steps. The positions of the high-symmetry points are indicated by dotted lines. The gray line is described in the text.

$\mathrm{Pb} / \mathrm{Ag}$ alloys at single crystal $\mathrm{Ag}$ surfaces and have been reported to be largely spin-split. ${ }^{34,35}$ They cross the Fermi level at 3-4 degrees off-normal and the Fermi wave number $k_{F}$ is $0.14 \AA^{-1}$, as shown by the white arrowhead. The para-
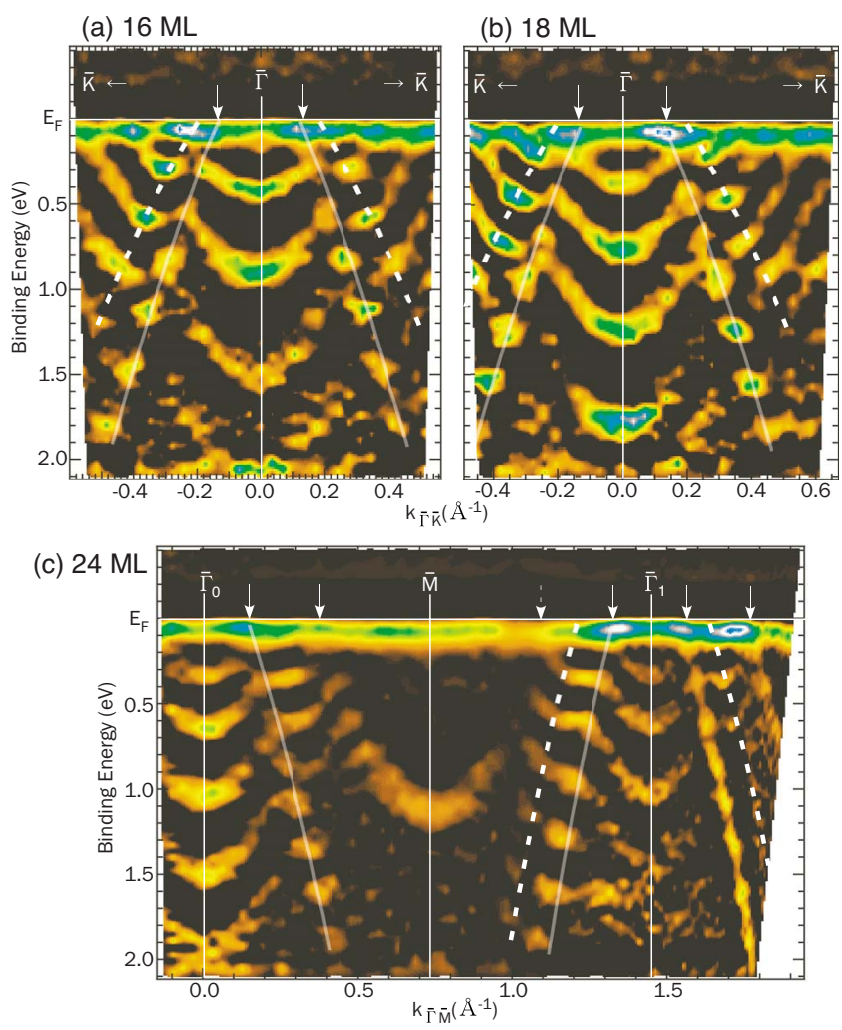

FIG. 4. (Color online) (a, b) The band dispersion image along the $\bar{\Gamma}-\bar{K}$ direction for the 16 and $18 \mathrm{ML} \operatorname{Ag}(111) \sqrt{3} \times \sqrt{3}-\mathrm{Pb}$ films. (c) The band dispersion image along the $\bar{\Gamma}-\bar{M}-\bar{\Gamma}$ direction for the 24 $\operatorname{ML~} \operatorname{Ag}(111) \sqrt{3} \times \sqrt{3}-\mathrm{Pb}$ film. The arrowheads show the Fermi-level crossings. The solid and dotted lines show the possible dispersion of the surface-state bands (see text). (a)
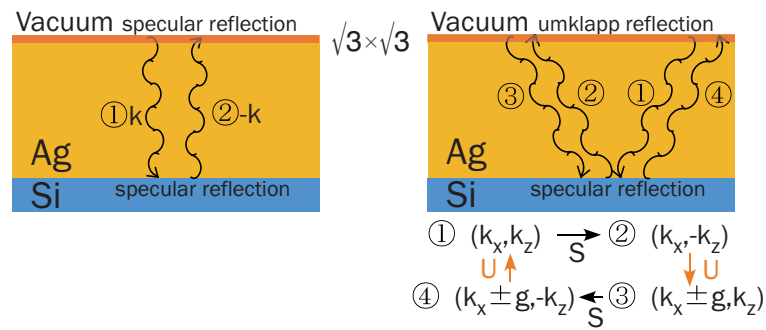

(b)
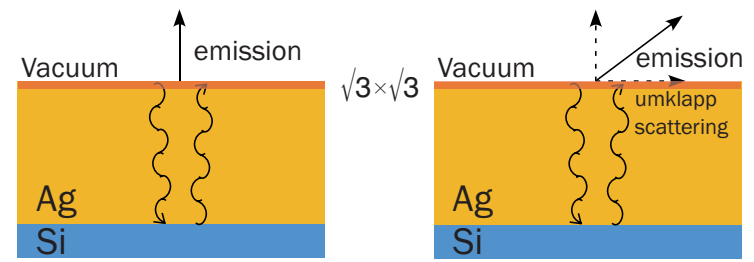

FIG. 5. (Color online) Schematic illustration of the initial- (a) and final- (b) state effects for the explanation of the quantum-well states observed at the $\bar{\Gamma}_{1}$ point of the $\sqrt{3} \times \sqrt{3}$ surface Brillouin zone.

bolic dispersion of the QWSs seem to be disturbed where they intersect with the surface states possibly due to hybridization effects. ${ }^{36}$ This may transfer some spin polarization to the QWSs at these crossing points. In Ref. 34, another Fermi-level crossing surface state very close to the one described above (see Fig. 6) was observed, but in our measurement they could not be detected that clearly. The peak intensities of these states are reported to be very weak compared to the ones we have observed ${ }^{34}$ and we speculate that they are lost due to the strong spectral intensity of the QWSs. However we believe that the intensity of the QWSs increases where they intersect with this surface-state band, and we can see its dispersion as shown by the dotted lines in Figs. 4(a) and 4(b). Also, some indication of these states can be found at the second Brillouin zone near $\bar{\Gamma}_{1}$ shown by the solid line in Fig. 3(c) and the dotted line in Fig. 4(c).

In Fig. 4(c), we also notice that there is another parabolic band around the $\bar{M}$ point with $k_{F} \sim 0.37 \AA^{-1}$, which was also assigned to be a surface state. ${ }^{27,34}$ Furthermore, we find that there are also quantum-well state bands at $\bar{\Gamma}_{1}$. Usually the QWSs on clean $\operatorname{Ag}(111)$ films obey the $1 \times 1$ periodicity and there are no spectral features at this $\bar{\Gamma}_{1}$ point which corresponds to the $\bar{K}$ point of $1 \times 1$. In the present case, the film itself has only the $1 \times 1$ periodicity, but due to the alloying, a $\sqrt{3} \times \sqrt{3}$ periodicity is present at the surface. There are two possible explanations for this observation, as schematically shown in Fig. 5. By assuming a two-dimensional model $(x$ : parallel to the surface, $z$ : normal to the surface), we can write the original wave vector as $\left(k_{x}, k_{z}\right)$. The usual quantum-well states are formed as a result of two consecutive specular $(S)$ reflections at the $\mathrm{Si} / \mathrm{Ag}$ and $\mathrm{Ag} /$ vacuum interfaces [left-hand side of Fig. 5(a)]. The QWSs at $\bar{\Gamma}_{1}$ can be thought of as due to four reflections: Starting from $\left(k_{x}, k_{z}\right)$, the electrons undergo a $S$ reflection at the $\mathrm{Si} / \mathrm{Ag}$ interface and become $\left(k_{x}\right.$, $-k_{z}$ ). At the $\mathrm{Ag} /$ vacuum interface, they undergo a umklapp $(U)$ reflection due to the $\sqrt{3} \times \sqrt{3}$ periodicity and the wave vector is now $\left(k_{x} \pm g, k_{z}\right)$. They are again $S$ reflected at the 

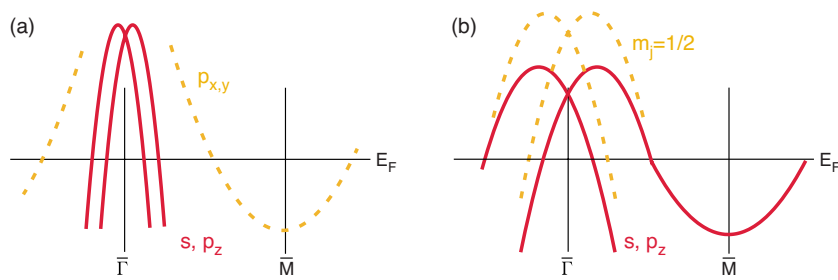

(c)

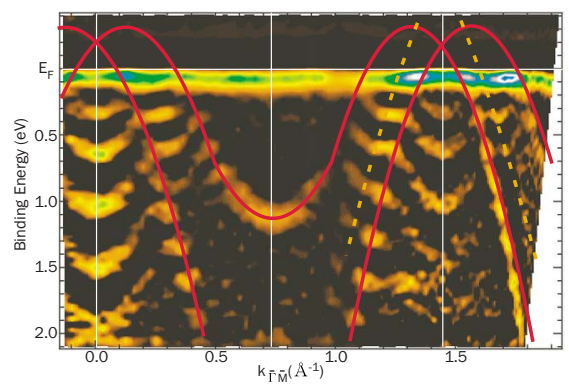

FIG. 6. (Color online) Schematic drawing of the band dispersion of the spin-orbit-split surface states as illustrated in Ref. 34 (a) and Ref. 27 (b), respectively. (c) Same as Fig. 4(c), but with the fitting based on the schematic drawing of (b) overlapped.

$\mathrm{Si} / \mathrm{Ag}$ interface $\left(k_{x} \pm g,-k_{z}\right)$ and finally return to their original wave vector after another $U$ reflection at the $\mathrm{Ag} /$ vacuum interface [right-hand side of Fig. 5(a)]. Such a model has been used to explain the QWSs observed at the $\bar{M}$ point of $\mathrm{Ag}$ films on $\mathrm{Ge}(111) .{ }^{15}$ We will call this an initial-state effect. In the second scenario, we just say that what is really present are the QWSs at the $\bar{\Gamma}_{0}$ point. They are emitted into the vacuum after the photoexcitation process and can be seen at $\bar{\Gamma}_{0}$ [left-hand side of Fig. 5(b)]. However, due to the strong potential at the surface (Ag/vacuum interface) which obeys the $\sqrt{3} \times \sqrt{3}$ periodicity, the photoemitted electrons are umklapp scattered and can also be observed at the $\bar{\Gamma}_{1}$ point [right-hand side of Fig. 5(b)]. In this sense, we call this a final-state effect. Whether the observed photoemission spectra is due to the initial- or final-state effect has been debated for several systems such as the $\operatorname{Si}(111) \sqrt{21} \times \sqrt{21}-\mathrm{Ag}$, -(Ag,Au) surfaces ${ }^{37,38}$ or $\mathrm{Si}(111) \sqrt{7} \times \sqrt{3}-\mathrm{In},{ }^{39}$ but it has been difficult to determine which is correct. Similarly, it is also difficult to conclude which of the effects is the reason for the presently observed $\sqrt{3} \times \sqrt{3}$ periodicity of the QWSs. ${ }^{40}$

Finally, we comment on the Rashba splitting of the surface-state bands. In Ref. 34, the authors concluded from ARPES and first-principles calculation without taking SOC into account that the two Fermi-level crossing bands near $\bar{\Gamma}$ are Rashba-split bands with another surface-state band dispersing near $\bar{M}$, as schematically shown in Fig. 6(a) with the orbital components explicitly written. The splitting was shown to be relatively small $\left(\Delta k=0.03 \AA^{-1}\right)$ compared to the similar $\mathrm{Bi} / \mathrm{Ag}(111)$ alloy $\left(\Delta k=0.13 \AA^{-1}\right){ }^{22}$ However,
Bihlmayer et al. have shown from ab initio calculations considering the SOC that the splitting is not that much different in the $\mathrm{Pb} / \mathrm{Ag}$ alloy $\left(\Delta k=0.11 \AA^{-1}\right)$ as compared to the $\mathrm{Bi} / \mathrm{Ag}$ alloy. They showed that there are two pairs of Rashba-split bands and the two bands that are close to the $\bar{\Gamma}$ point are composed of different orbitals [Fig. 6(b)]. As has been discussed above, we could not observe the metallic surface-state band that is the second closest to $\bar{\Gamma}$ that clearly. Considering that the photoemission intensity or the matrix element effects should not be that different for states that are composed of the same orbitals near normal emission, we take the latter view in our discussion. If we make a fitting to the surfacestate bands of Fig. 4(c) using the dispersion shown in Fig. 6(b), we come up with $\Delta k \sim 0.12 \AA^{-1}$, Rashba energy $E_{R}$ $=\hbar^{2} \Delta k^{2} /\left(2 m^{*}\right) \sim 120 \mathrm{meV}$, and Rashba parameter $\alpha_{R}$ $=\hbar^{2} \Delta k / m^{*} \sim 2 \mathrm{eV} \AA$ [Fig. 6(c)] for the $s, p_{z}$ band. The value of $\Delta k$ is considerably larger than that reported in Ref. 34, but $\alpha_{R}$ is nearly similar (Ref. 34 shows $\alpha_{R}=1.42 \mathrm{eV} \AA$ ), although still smaller than $\mathrm{Bi} / \mathrm{Ag}\left(\alpha_{R}=3.05 \mathrm{eV} \AA\right)$. For the $m_{j}=1 / 2$ band, although it is quite difficult to deduce the correct value from our data, we obtain $\Delta k \sim 0.08 \AA^{-1}$ and $\alpha_{R} \sim 1.3 \mathrm{eV} \AA$. Even though the recent spin-resolved ARPES measurements suggest that the scenario of Fig. 6(a) is preferable,$^{41}$ the spin polarization analyses in Ref. 27 show that the behavior of the spin orientation is quite complex and the experimental observation does not necessarily contradict Fig. 6(b). Further extensive work is needed to distinguish the real situation.

\section{CONCLUSIONS}

In conclusion, we have performed ARPES measurements on ultrathin Ag films whose surface is alloyed with 1/3 ML $\mathrm{Pb}$. Due to the change in the phase shift at the film/vacuum interface because of the stronger confinement, the energy levels of the QWSs moved closer to the Fermi level. The QWSs obeyed the $\sqrt{3} \times \sqrt{3}$ periodicity that is only present at the surface, possibly due to umklapp reflection at the film/ vacuum interface or the umklapp scattering in the photoemission process. We have also discussed the Rashba splitting of the surface-state bands. Our results show that it is possible to manipulate the properties of the QWSs in ultrathin films by depositing submonolayer of hetero elements and making a surface alloy. It would be interesting to investigate how the spin properties of the QWSs are changed where they hybridize with the surface states. ${ }^{36}$

\section{ACKNOWLEDGMENTS}

We acknowledge K. Takase for her help in the ARPES measurement and T. Nagao for his valuable discussion. This work was supported by Grants-In-Aid from Japanese Society for the Promotion of Science. 
*hirahara@surface.phys.s.u-tokyo.ac.jp

${ }^{1}$ T.-C. Chiang, Surf. Sci. Rep. 39, 181 (2000).

${ }^{2}$ I. Matsuda, H. W. Yeom, T. Tanikawa, K. Tono, T. Nagao, S. Hasegawa, and T. Ohta, Phys. Rev. B 63, 125325 (2001); I. Matsuda, T. Tanikawa, S. Hasegawa, H. W. Yeom, K. Tono, and T. Ohta, e-J. Surf. Sci. Nanotechnol. 2, 169 (2004).

${ }^{3}$ T. Hirahara, T. Nagao, I. Matsuda, G. Bihlmayer, E. V. Chulkov, Yu. M. Koroteev, and S. Hasegawa, Phys. Rev. B 75, 035422 (2007).

${ }^{4}$ N. V. Smith, Phys. Rev. B 32, 3549 (1985).

${ }^{5}$ F. Patthey and W.-D. Schneider, Phys. Rev. B 50, 17560 (1994).

${ }^{6}$ M. A. Mueller, T. Miller, and T.-C. Chiang, Phys. Rev. B 41, 5214 (1990).

${ }^{7}$ J. E. Ortega, F. J. Himpsel, G. J. Mankey, and R. F. Willis, Surf. Rev. Lett. 4, 361 (1997); Phys. Rev. B 47, 1540 (1993).

${ }^{8}$ D. Wegner, A. Bauer, and G. Kaindl, Phys. Rev. Lett. 94, 126804 (2005).

${ }^{9}$ S. Ogawa, S. Heike, H. Takahashi, and T. Hashizume, J. Appl. Phys. 101, 09G504 (2007).

${ }^{10}$ Y. Guo, Y.-F. Zhang, X.-Y. Bao, T.-Z. Han, Z. Tang, L.-X. Zhang, W.-G. Zhu, E. G. Wang, Q. Niu, Z. Q. Qiu, J.-F. Jia, Z.-X. Zhao, and Q.-K. Xue, Science 306, 1915 (2004).

${ }^{11}$ Y.-S. Fu, S.-H. Ji, X. Chen, X.-C. Ma, R. Wu, C.-C. Wang, W.-H. Duan, X.-H. Qiu, B. Sun, P. Zhang, J.-F. Jia, and Q.-K. Xue, Phys. Rev. Lett. 99, 256601 (2007).

${ }^{12}$ N. Miyata, K. Horikoshi, T. Hirahara, S. Hasegawa, C. M. Wei, and I. Matsuda (unpublished).

${ }^{13}$ I. Matsuda, T. Ohta, and H. W. Yeom, Phys. Rev. B 65, 085327 (2002).

${ }^{14}$ N. J. Speer, S.-J. Tang, T. Miller, and T.-C. Chiang, Science 314, 804 (2006).

${ }^{15}$ S.-J. Tang, Y.-R. Lee, S.-L. Chang, T. Miller, and T.-C. Chiang, Phys. Rev. Lett. 96, 216803 (2006).

${ }^{16}$ P. Moras, L. Ferrari, C. Spezzani, S. Gardonio, M. Ležaić, $\mathrm{Ph}$. Mavropoulos, S. Blügel, and C. Carbone, Phys. Rev. Lett. 97, 206802 (2006).

${ }^{17}$ P. Moras, W. Theis, L. Ferrari, S. Gardonio, J. Fujii, K. Horn, and C. Carbone, Phys. Rev. Lett. 96, 156401 (2006).

${ }^{18}$ N. Nagamura, I. Matsuda, N. Miyata, T. Hirahara, S. Hasegawa, and T. Uchihashi, Phys. Rev. Lett. 96, 256801 (2006).

${ }^{19}$ T. Hirahara, T. Nagao, I. Matsuda, G. Bihlmayer, E. V. Chulkov, Yu. M. Koroteev, P. M. Echenique, M. Saito, and S. Hasegawa, Phys. Rev. Lett. 97, 146803 (2006).

${ }^{20}$ J. I. Pascual, G. Bihlmayer, Yu. M. Koroteev, H.-P. Rust, G. Ceballos, M. Hansmann, K. Horn, E. V. Chulkov, S. Blügel, P. M. Echenique, and Ph. Hofmann, Phys. Rev. Lett. 93, 196802 (2004).

${ }^{21}$ T. Hirahara, K. Miyamoto, I. Matsuda, T. Kadono, A. Kimura, T. Nagao, G. Bihlmayer, E. V. Chulkov, S. Qiao, K. Shimada, H. Namatame, M. Taniguchi, and S. Hasegawa, Phys. Rev. B 76, 153305 (2007).
${ }^{22}$ C. R. Ast, J. Henk, A. Ernst, L. Moreschini, M. C. Falub, D. Pacilé, P. Bruno, K. Kern, and M. Grioni, Phys. Rev. Lett. 98, 186807 (2007).

${ }^{23}$ As reported in Ref. 2, the surface states and QWSs are not affected by this annealing for the clean $\mathrm{Ag}(111)$ films.

${ }^{24}$ T. Hirahara, I. Matsuda, M. Ueno, and S. Hasegawa, Surf. Sci. 563, 191 (2004).

${ }^{25}$ M. Hupalo, T. L. Chan, C. Z. Wang, K. M. Ho, and M. C. Tringides, Phys. Rev. B 66, 161410(R)(2002).

${ }^{26}$ C. Li, A. J. Freeman, H. J. F. Jansen, and C. L. Fu, Phys. Rev. B 42, 5433 (1990).

${ }^{27}$ G. Bihlmayer, S. Blügel, and E. V. Chulkov, Phys. Rev. B 75, 195414 (2007).

${ }^{28}$ P. M. Echenique and J. B. Pendry, J. Phys. C 11, 2065 (1978).

${ }^{29}$ Although the $\mathrm{Pb}$ deposition will increase the film thickness by $1 / 3 \mathrm{ML}$, we have basically neglected this effect in the analyses because the film thickness itself already has uncertainties that are reflected in the error bars in Figs. 2(a) and 2(b). We believe that the basic physical picture will not be altered even if the film thickness may not be precise.

${ }^{30}$ E. G. McRae and M. L. Kane, Surf. Sci. 108, 435 (1981).

${ }^{31}$ K. J. Rawlings, M. J. Gibson, and P. J. Dobson, J. Phys. D 11, 2059 (1978).

${ }^{32}$ K. Takayanagi, D. M. Kolb, K. Kambe, and G. Lehmpfuhl, Surf. Sci. 104, 527 (1981).

${ }^{33}$ Q. Yan, J. Wu, G. Zhou, W. Duan, and B. L. Gu, Phys. Rev. B 72, 155425 (2005).

${ }^{34}$ D. Pacilé, C. R. Ast, M. Papagno, C. Da Silva, L. Moreschini, M. Falub, A. P. Seitsonen, and M. Grioni, Phys. Rev. B 73, 245429 (2006).

${ }^{35}$ C. R. Ast, D. Pacilé, M. Papagno, T. Gloor, F. Mila, S. Fedrigo, G. Wittich, K. Kern, H. Brune, and M. Grioni, Phys. Rev. B 73, 245428 (2006).

${ }^{36}$ K. He, T. Hirahara, T. Okuda, S. Hasegawa, A. Kakizaki, and I. Matsuda (unpublished).

${ }^{37}$ I. Matsuda, T. Hirahara, M. Konishi, C. Liu, H. Morikawa, M. Dangelo, S. Hasegawa, T. Okuda, and T. Kinoshita, Phys. Rev. B 71, 235315 (2005).

${ }^{38}$ J. N. Crain, K. N. Altmann, C. Bromberger, and F. J. Himpsel, Phys. Rev. B 66, 205302 (2002).

${ }^{39}$ E. Rotenberg, H. Koh, K. Rossnagel, H. W. Yeom, J. Schäfer, B. Krenzer, M. P. Rocha, and S. D. Kevan, Phys. Rev. Lett. 91, 246404 (2003).

${ }^{40}$ The QWSs observed at off-normal in Refs. 15 and 16 can generally be considered as an initial-state effect because the periodicity comes from the film/substrate interface and it is unlikely that photoemitted electrons will feel this potential at the interface when they go out into the vacuum.

${ }^{41}$ F. Meier, H. Dil, J. Lobo-Checa, L. Patthey, and J. Osterwalder, Phys. Rev. B 77, 165431 (2008). 